\title{
Diacronie
}

Studi di Storia Contemporanea

$\mathrm{N}^{\circ} 25,1 \mid 2016$

"Se creare è definire"

\section{«Federarsi o perire». Il contributo britannico al federalismo europeo}

\section{Benedetta Giuliani}

\section{(C) OpenEdition}

\section{Journals}

\section{Edizione digitale}

URL: http://journals.openedition.org/diacronie/3944

DOI: $10.4000 /$ diacronie.3944

ISSN: 2038-0925

\section{Editore}

Association culturelle Diacronie

\section{Notizia bibliografica digitale}

Benedetta Giuliani, « «Federarsi o perire». Il contributo britannico al federalismo europeo », Diacronie [Online], N 25, 1 | 2016, documento 5, Messo online il 29 mars 2016, consultato il 03 mai 2019. URL : http://journals.openedition.org/diacronie/3944 ; DOI : 10.4000/diacronie.3944 


\title{
Diacronie
}

\section{$5 /$}

\section{«Federarsi o perire»}

\section{Il contributo britannico al federalismo europeo}

\author{
Benedetta GIULIANI *
}

Trincerata nel mito di un isolamento dorato che sembrava destinato a perdurare anche davanti alle profonde alterazioni degli equilibri globali in atto nel XX secolo, la Gran Bretagna è stata tradizionalmente considerata estranea ai progetti di integrazione politica del Vecchio continente. Eppure fu proprio la cultura politica inglese ad offrire un significativo contributo allo sviluppo del pensiero europeista e nello specifico alla sua declinazione federalista. L'articolo intende esaminare il rapporto tra la Gran Bretagna e la causa dellintegrazione europea, con una particolare attenzione al gruppo di Federal Union attivo nel biennio 1938-40 per l'instaurazione di una federazione europea.

\section{La Gran Bretagna tra isolazionismo ed europeismo}

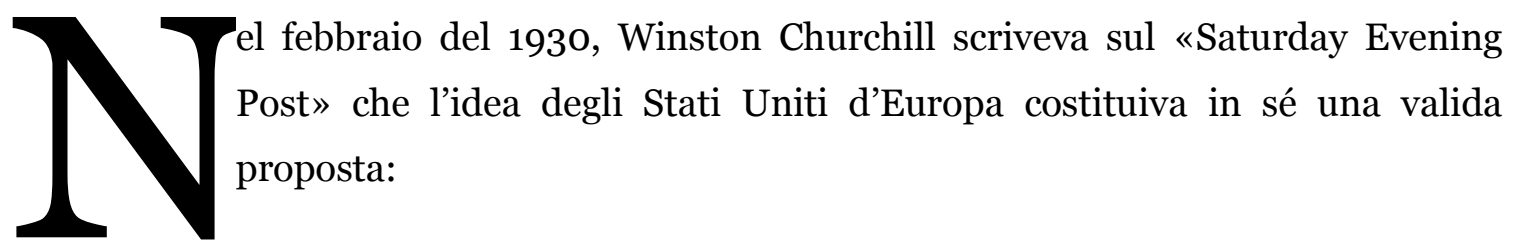

Every step taken to that end which appeases obsolete hatreds and vanished oppressions, which makes easier the traffic and reciprocal services of Europe [...] is good in itself1.

Nonostante il giudizio positivo circa l'istituzione di una forma di governo unificato europeo, lo statista inglese, nel medesimo articolo, si preoccupò di specificare che la Gran Bretagna, in virtù della propria vocazione di potenza non europea ma mondiale,

${ }^{1}$ CHURCHILL, Winston, «The United States of Europe», in Saturday Evening Post, 15 febbraio 1930. 
sarebbe stato un alleato piuttosto che un membro a pieno titolo, della eventuale federazione europea. La Gran Bretagna, nelle parole di Churchill e nel pensiero di molti suoi illustri colleghi, poteva schierarsi con l'Europa ma non poteva essere parte di essa. Sarebbe forse sufficiente studiare l'approccio di Churchill alla questione europea, tra il 1946 e il 1951, per comprendere la prospettiva "anglo-centrica" con cui la Gran Bretagna guardava al Vecchio continente. Churchill, pur facendosi promotore dell'unificazione europea (prima con il celebre discorso pronunciato a Zurigo nel 1946, in cui non esitò a dirsi favorevole agli «Stati Uniti d'Europa»², poi con la creazione del Movimento per l'Europa Unita nel 1947), una volta tornato al governo nel 1951 dimostrò di non aver rinnegato il consueto modus operandi nei confronti dell'Europa. Ovvero controllare da vicino i rapporti di forza nel continente, onde evitare l'egemonia eversiva di qualche Stato rivale, senza tuttavia rinunciare al proprio status di potenza imperiale che considerava l'area del Commonwealth e il resto dello English-speakingWorld quali sfere d'azione privilegiati ${ }^{3}$. Affidando la tutela della propria sicurezza esterna a una prassi che combinava il mantenimento dell'equilibrio tra potenze con il principio di non intervento nelle dispute europee, la Gran Bretagna si garantiva un ruolo da potenza super partes, capace di intervenire in sede diplomatica per ostacolare l'emergere di una forza predominante sul continente, fosse essa rappresentata da una singola potenza o da una coalizione di Stati ${ }^{4}$. Tale atteggiamento, ispirato più alla strategia del divide et impera che a una politica di integrazione, indirizzò la politica estera britannica fino al 1914.

Invero, se volessimo ripercorrere la storia della coscienza di una comune identità tra i popoli europei, dovremmo ammettere che essa maturò tardivamente in tutto il continente, non solo in Gran Bretagna. Se ai britannici è stato imputato di essere un popolo, per usare una terminologia moderna, di euroscettici, bisogna pur riconoscere

\footnotetext{
2 Il discorso di Zurigo rilanciò il dibattito pubblico sull'unità europea nel dopoguerra. In quel contesto Churchill affermò che la riconciliazione tra Francia e Germania, indispensabile per la pacificazione europea, si sarebbe potuta raggiungere tramite una struttura rappresentativa sovranazionale, che egli identificò con gli Stati Uniti d'Europa. La strategia europeista di Churchill finì tuttavia col promuovere la creazione di un concerto di Stati sovrani, quale fu il Consiglio d'Europa progettato nel 1948. Cfr. ANTA, Claudio Giulio, The Europeanism of Winston Churchill, Roma, Aracne Editrice, 2009, pp. 30-31; CHURCHILL, Winston, Address given by Winston Churchill at the Congress of Europe in The Hague (7 may 1948), URL: < http://www.cvce.eu/obj/address_given_by_winston_churchill_at_the_congress_of_europe_i n_the_hague_7_may_1948-en-58118da1-af22-48co-bc88-93cda974f42c.html $>$ [consultato il 2 giugno 2014].

3 CHURCHILL, Winston, Conservative Mass Meeting. A Speech at Llandudno, in ID., Europe Unite: Speeches 1947 \& 1948, London, Cassell, 1950, pp. 416-418, URL: < http://webarchives.univ-pau.fr/english/special/SRdoc1.pdf > [consultato il 22 febbraio 2016].

4 WILMOT, Chester, «Britain's Strategic Relationship to Europe», in International Affairs, 29, 4/1953, pp. 409-417.
} 
che anche altri Paesi europei non ebbero la capacità o la volontà di promuovere manifestazioni di carattere pubblico di valore in favore dell'unità europea. Nell'orizzonte post-1918 uniche eccezioni di rilievo sembrano essere il Movimento Paneuropa, fondato nel 1923 dal conte asburgico Richard Coudenhove-Kalergi, per un'unione (Staatenbund) europea 5 , e il progetto di una custom union suggerito dal ministro degli esteri di Francia Aristide Briand nel 1929, quest'ultimo espressione di un approccio gradualistico all’integrazione che non intaccasse la sovranità degli Statíb. I progetti di Kalergi e Briand riuscirono a imporsi all'attenzione dell'opinione pubblica europea sfruttando la temperie culturale della metà degli anni Venti, sollecitata dalle crisi recenti ad elaborare una critica dello Stato-nazione e dell'anarchia internazionale che da esso derivava; tuttavia la loro capacità di sensibilizzare i governi sul tema della cooperazione sovranazionale fu limitata, o a causa di mancanze intrinseche (il carattere elitario e la fumosa retorica escatologica dell'iniziativa di Kalergi) o di congiunture storiche sfavorevoli (l'esaurirsi della fase di distensione diplomatica instaurata dagli accordi franco-tedeschi di Locarno, nel caso del Piano Briand).

L'idea di un progetto istituzionale europeo divenne un obiettivo perseguito dai governi solo nel 1945, davanti alla realtà di un continente ridotto alla subalternità politica e finanziaria in seguito all'affermazione del duopolio Stati Uniti-Unione Sovietica. Ma se l'apparato dello Stato-nazione appariva ormai inservibile agli sconfitti, come Italia e Germania, altrettanto non si poteva dire della Gran Bretagna, ancora convinta di essere "al centro dell'Europa", forte della superiorità morale che le derivava dal ruolo di primo piano svolto nella guerra contro il nazismo7. Anziché le debolezze strutturali, la guerra sembrava aver esaltato anche nelle avversità del conflitto le infinite potenzialità dello Stato-nazione ${ }^{8}$. L'elusiva accoglienza di Londra nei confronti dei progetti di integrazione europea spesso percepiti o presentati, da una variegata schiera di esponenti politici, come estranei all'ethos della nazione britannica, continuava ad essere una costante della storia, nonché una tendenza trasversale allo spettro delle forze governative. Non sembra casuale, infatti, la somiglianza tra

5 Sulle iniziative federaliste di Kalergi si vedano COUDENHOVE-KALERGI, Richard, Paneuropa, 1922 bis 1966, Wien, Herold, 1966; GUI, Francesco, «Una costituzione per l'Europa d'altri tempi, ma con preveggenze d'attualità. Il testo del progetto del 6 maggio 1951 del Conte Coudenhove-Kalergi», in Eurostudium, 35/2015, pp. 41-52.

${ }^{6}$ FREYMOND, Jean, «Gustav Stresemann et l'idée d'une "Europe économique" (1925-1927)», in Relations Internationales, 8/1976, pp. 343-360; NAVARI, Cornelia, The Origins of the Briand Plan, in BOSCO, Andrea (ed.), The Federal Idea, vol. I, London, Lothian Foundation Press, 1991, pp. 211-236.

7 GILBERT, Michael, European Integration. A Concise History, Lanham, Rowman and Littlefield, 2011, p. 11.

8 BOGDANOR, Vernon, «Footfalls Echoing In The Memory. Britain and Europe. The Historical Perspective», in International Affairs, 81, 4/2005, pp. 689-701. 
l'affermazione del tory Anthony Eden, secondo cui la Gran Bretagna non poteva compromettersi in un'unione con l'Europa occidentale, date le ambizioni mondialistiche della nazione britannica9 ${ }^{9}$ e quella del laburista Hugh Gaitskell secondo cui l’ingresso della Gran Bretagna nella CEE avrebbe messo a repentaglio «mille anni di storia» ${ }^{10}$. L'atteggiamento istituzionale con cui la Gran Bretagna accolse l’integrazione europea, fatto di diffidenza e volontà di difendere un glorioso passato nazionale, si configura come la difesa, a un tempo politica e culturale, di due concetti che innervano l'identità storica anglosassone. In primo luogo quello di impero, simbolo di forza e indipendenza cui la Gran Bretagna, ancora nel dopoguerra, resterà attaccata nella convinzione che quest'ultimo nella forma rinnovata del Commonwealth of Nations (assunto quale fulgido esempio di Stato trans-nazionale, in grado di conciliare autonomia locale e sovranazionalità ${ }^{11}$ ) potesse sfuggire la decadenza toccata in sorte ad altri imperi coloniali. In secondo luogo, quello di sovranità parlamentare che si configura come una categoria interpretativa capitale per la comprensione della storia politica inglese:

The political regime in Britain has remained basically unchanged since 1689, the year of the Glorious Revolution [...] which emphasized the undivided sovereignty of Parliament ${ }^{12}$.

La possibilità di cedere anche solo parzialmente la propria sovranità per ripartirla a un livello sovranazionale appariva ai britannici come un rischioso tentativo di compromettere una stabilità secolare per un coinvolgimento negli affari di un continente la cui storia recente, a differenza di quella inglese, continuava ad essere scandita da crisi rivoluzionarie ${ }^{13}$. Tuttavia, un recente filone della storiografia britannica ${ }^{14}$, nell'indagare le strategie di risposta della Gran Bretagna verso gli eterogenei progetti di integrazione europea, i quali iniziano ad emergere con continuità tra il 1789 e il 1814, ha dimostrato che queste non possono essere descritte solo in

\footnotetext{
9 EDEN, Anthony, «Great Britain, Europe and the Commonwealth», in Le Monde, 24 dicembre 1949.

10 GAITSKELL, Hugh, citato in GILBERT, Michael, op. cit., p. 73.

${ }^{11}$ Sulle interpretazioni dell'impero britannico come soggetto storico eccezionale ed ecumenico si veda TAGLIAFERRI, Teodoro, Dalla Greater Britain al World Order, in ROMANELLI, Raffaele, Impero, imperi. Una conversazione, Napoli-Roma, L'Ancora del Mare, 2009, pp. 187217.

12 BOGDANOR, Vernon, op. cit., p. 697.

13 Ibidem.

${ }_{14}$ Cfr. BURGESS, Michael, The British Tradition of Federalism, London, Leicester University Press, 1995; ID., Comparative Federalism. Theory and Practice, London, Routledge, 2006, pp. 177-185; KENDLE, John, Federal Britain. A History, London, Routledge, 1997.
} 
termini di scostante ostilità. Si può anzi rintracciare un rapporto prolungato, benché contraddittorio e problematico, tra la cultura politica britannica e l'idea di integrazione europea. Occorrerà sottolineare che il modello di integrazione preso in considerazione è quello federale secondo cui, per assicurare una coesistenza pacifica tra gli Stati europei, è necessario che gli ambiti fondamentali dell'azione di governo (politica estera, monetaria, etc.) debbano essere sottratti al controllo dei singoli Stati - con una conseguente limitazione della loro sovranità - e affidati a organismi politici comuni ${ }^{15}$.

Come rivelato dagli studi di Daniele Archibugi e Franco Voltaggio ${ }^{16}$, l'impegno per la costruzione di una architettura governativa sovranazionale comparve straordinariamente presto nella teoria politica inglese, benché inizialmente circoscritto a gruppi minoritari di radicali pacifisti e quaccheri ${ }^{17}$, la cui principale preoccupazione era quella di individuare una strategia che permettesse di appianare le dispute tra Stati sovrani senza il ricorso alle armi. Tale attitudine europeista crebbe e maturò nel tempo, guadagnando lentamente consensi presso il ceto dirigente britannico ${ }^{18}$. Nel 1871 spettò al professore di Storia Moderna John Seeley pronunciare, per conto della Peace Society, un intervento ${ }^{19}$ in cui rivendicava la necessità di instaurare una federazione europea di Stati, modellata su quella americana. La proposta di Seeley risultava innovativa nella misura in cui combinava topoi ricorrenti nel dibattito accademico britannico sul federalismo (ad esempio, il riferimento alla nuova forma di Stato nata in America quale archetipo di riferimento) con aspetti di spiccata modernità. Seeley tendeva a escludere le corti di arbitrato come assemblee capaci di assicurare un assetto europeo pacificato, sostenendo piuttosto la creazione di una "vera unione di popoli" nata da un "generale movimento popolare". La costruzione di un sistema federale europeo veniva perciò considerata non come il frutto di una rivoluzione politica guidata dall'alto, bensì come l'esito di una nuova coscienza patriottica, non più nazionalistica ma europea ${ }^{20}$. La disamina di Seeley giunse a considerare il federalismo come un dispositivo giuridico

${ }^{15} \mathrm{Si}$ veda la voce "Europeismo" redatta da Altiero Spinelli per l'Enciclopedia del Novecento, vol. II, Roma, Istituto dell'Enciclopedia Italiana, 1977.

${ }^{16}$ ARCHIBUGI, Daniele, VOLTAGGIO, Franco, Filosofi per la Pace, Roma, Editori Riuniti, 1991, pp. XXII, XXXVI.

${ }_{17}$ Risale al 1693 il primo progetto per la costruzione di un Parlamento europeo composto da deputati scelti tra i cittadini, avanzata dal quacchero William Penn. Cfr. PENN, William, An Essay Towards the Present and Future Peace of Europe, Washington, The American Peace Society, 1912, pp. 3 et seq.; GUCCIONE, Cristina, William Penn. Forme di governo e identità linguistica, Torino, Giappichelli, 2008, pp. 129-170.

${ }^{18}$ CEADEL, Martin, Supranationalism in the British Peace Movement, in BOSCO, Andrea (ed.), The Federal Idea, cit., p. 175.

19 SEELEY, John, «The United States of Europe. A Lecture delivered before the Peace Society», in Macmillan's Magazine, 23, 1870, pp. 436-448.

${ }^{20}$ ID., citato in BURGESS, Michael, Federalism and Federation in Western Europe, London, Croom Helm, 1986, pp. 139-140. 
atto a risolvere la conflittualità tra Stati nazionali, e attribuì al discorso federalista una dimensione pragmatica assente nelle indagini coeve sul modello federale, più interessate a ricercare una concettualizzazione storico-scientifica del fenomeno che non a considerarla quale concreta opzione politica ${ }^{21}$.

Il progetto di una federazione europea guadagnò notevole consenso nel corso del tormentato periodo che precedette lo scoppio della Prima guerra mondiale. In questo periodo furono create vere e proprie associazioni per la promozione di un'unione politica europea, tra cui si ricorderà la European Unity League, fondata dall'industriale Max Waecther, che ebbe scarsa risonanza politica ma coniugò, con mirabile anticipo, l'integrazione istituzionale e quella economica, promuovendo l'instaurazione di un mercato comune europeo ${ }^{22}$. Altrettanto importante fu il cambiamento culturale che indusse coloro che in Gran Bretagna avevano a cuore la stabilità del Vecchio continente a intraprendere un profondo processo di ripensamento dell'assetto politico europeo, nel tentativo di individuare le cause all'origine delle cicliche fasi di guerra europee.

È interessante notare il progressivo superamento delle concezioni tradizionali del conflitto. Se in precedenza la guerra era stata considerata o un male necessario (quasi che la caratteristica peculiare dei popoli fosse la tendenza a una violenza, per così dire, intra-specifica) o un legittimo strumento nella condotta della politica estera, tra il 1914 e il 1918 si iniziò a guardare ad essa come a una falla all'interno di un sistema di per sé mal funzionante: quello degli Stati-nazione. La sovranità nazionale, strutturando le entità statali alla stregua di monadi accentrate, separate tra di loro, iniziò ad essere considerata il principio di quel grave iato tra lo sviluppo dei rapporti economici e lo stato dei rapporti politici tra nazioni europee. Se infatti la moderna società industriale, giunta in una fase di maturità capitalistica, sembrava plasmare l'Europa come un insieme economicamente integrato, le relazioni politiche continuavano ad essere impostate secondo logiche di divisione e competizione tra Stati ${ }^{23}$. L'aporia tra la dimensione nazionale del potere politico e quella internazionale dello sviluppo economico (la condizione per cui esisteva un'economia mondiale ma non una politica

${ }^{21}$ Autori contemporanei di Seeley si dedicarono ad analizzare le origini storiche e le qualità giuridiche del federalismo, senza però ammetterne l'applicazione né nell'ambito nazionale britannico né, tantomeno, in un contesto sovranazionale europeo. Cfr. FREEMAN, Edward, History of Federal Government in Greece and Italy, London, Macmillan and Co., 1893, p. 70, URL: < https://archive.org./details/historyfederalgo2freegoog > [consultato il 19 febbraio 2016]; DICEY, Albert Venn, Introduction to the Study of the Law of the Constitution, London, Macmillan and Co., 1889, p. 129, URL:

$<$ https://archive.org/details/introductiontoso4dicegoog > [consultato il 19 febbraio 2016].

${ }^{22}$ WAECHTER, Max, «For United Europe, Not to Oppose Us», in The New York Times, 20 settembre 1908.

${ }^{23}$ LEVI, Lucio, «La crisi dello Stato nazionale e il problema dell'unificazione europea nell'epoca delle guerre mondiali», in The Federalist, 1/1975, pp. 4 et seq. 
mondiale, come sostenne l'economista Lionel Robbins ${ }^{24}$ ) venne individuata da alcuni intellettuali e funzionari di governo inglesi come la causa della persistente instabilità europea:

Sovereign nations-state in the absence of some kind of effective overarching international machinery to arbitrate peacefully between them, equated security simply with military strength. They were in a condition of perpetual rivalry and permanent conflict, which would intermittently, but inevitably, lead to war ${ }^{25}$.

Si verificò insomma il passaggio da un "internazionalismo morale" a un "internazionalismo istituzionale": non era la coscienza etica dell'uomo a dover mutare, bensì i rapporti di forza che il sistema delle sovranità nazionali aveva instaurato²6.

Fu dunque con il declinare dell'egemonia marittima britannica e con la catastrofe del 1914 che una parte della società britannica iniziò a considerare l’integrazione del continente su base federale come il dispositivo costituzionale adeguato per salvare l'Europa dal conflitto. Durante gli anni Venti del Novecento la corrente di pensiero europeista, fino ad allora presente con andamento carsico nella tradizione politica europea, emerse con rinnovato vigore in seguito all'incapacità dei governi di realizzare una cornice istituzionale entro la quale gli Stati potessero cooperare in pace. La neonata Società delle Nazioni, sorta attraverso un tribolato processo di negoziato durante la Conferenza di pace di Parigi, venne alla luce con i difetti congeniti tipici delle corti di arbitrato. Essa appariva, agli occhi dei sostenitori di una più solida integrazione europea, poco più di una commissione arbitrale troppo debole perché si potesse realizzare la sua trasformazione in un effettivo esecutivo sovranazionale. Trattandosi di una assise intergovernativa, secondo alcuni essa non era «nulla di più di quello che $\mathrm{i}$ governi, nelle capitali nazionali, vogliono che sia» ${ }^{27}$.

Nel corso degli anni Trenta i timori sull’inefficienza della Società delle Nazioni parvero confermarsi. Dotata di poteri essenzialmente consultivi e priva di una forza militare autonoma, la Società si dimostrò incapace di far fronte alle nuove crisi continentali, come le ripetute violazioni del Trattato di Versailles da parte della Germania hitleriana. Davanti all'impotenza della Società delle Nazioni iniziò a diffondersi, tanto nella società quanto nell'establishment britannico, il desiderio di

\footnotetext{
24 MONTANI, Guido (a cura di), «Lionel Robbins», in The Federalist, 2/1984, pp. 165-180. 25 BURGESS, Michael, The British Tradition of Federalism, cit., p. 135.

26 ALDOBRANDINI, Giovanni, The Wishful Thinking. Storia del pacifismo inglese nell'Ottocento, Roma, Luiss University Press, 2009, p. 517.

${ }_{27}$ KERR, Philip, Il Pacifismo non basta, Bologna, Il Mulino, 1986, p. 53.
} 
un'istituzione internazionale diversa, autorevole e dotata di un potere decisionale da opporre ai singoli Stati ${ }^{28}$. Fu questo insieme di paure e speranze ad incoraggiare la riscossa dell'iniziativa europeista.

\section{Federal Union e gli Stati Uniti d'Europa}

Federal Union, la prima associazione politica a centrare il focus della propria azione sulla creazione di un sistema parlamentare europeo eletto per via democratica, venne fondata nell'autunno del 1938. Charles Kimber, uno dei fondatori, descriveva così le circostanze che portarono alla nascita dell'organizzazione federalista:

Federal Union fu figlia degli anni Trenta. [...] Quattro nazioni europee avevano effettivamente il potere di mantenere la pace o di scatenare un'altra guerra mondiale. Quelli che erano sopravvissuti a quello squallido e tuttavia eroico massacro o che, come noi, erano cresciuti nel dopoguerra, avevano creduto che doveva veramente essere stata la guerra che avrebbe messo fine a tutte le guerre, $\mathrm{e}$ avevano posto la loro fiducia nella Società delle Nazioni. [...] Già verso la metà degli anni Trenta l'avevamo vista deliberatamente sabotare da tutte e quattro le nazioni. [...] Col passare dei mesi e degli anni si scivolava sempre più velocemente verso la guerra. Nessuno la voleva, eppure sembrava non esservi rimedio. Fu in questa atmosfera che fu ideata Federal Union 29.

L’istituzione del movimento si dovette all'iniziativa di due ex studenti di Oxford, Derek Rawnsley e Charles Kimber ${ }^{30}$. Rawnsely e Kimber, al tempo impiegati presso una compagnia petrolifera, iniziarono a dibattere la situazione europea allora dominata dall'aggressività tedesca che aveva aperto la crisi cecoslovacca, e finirono col coinvolgere in quei dibattiti anche altri colleghi ${ }^{31}$. Fu Rawnsley a suggerire a Kimber di conferire a quegli incontri una cornice ufficiale e un preciso programma d'azione. L’inefficienza della Società delle Nazioni, una "assemblea di unità indipendenti” in balia degli interessi particolari dei propri membri, rappresentò per tutti coloro che militarono in Federal Union il paradigma del fallimento della cooperazione tra Stati

28 BOSCO, Andrea, Lord Lothian and the creation of Atlantic Policy 1882-1940, London, Lothian Foundation Press, 2012, pp. 280-281.

${ }^{29}$ KIMBER, Charles, «La Nascita di Federal Union», in The Federalist, 3/1984, pp. 206-213.

$3^{3}$ BURGESS, Michael, The British Tradition of Federalism, cit., p. 140.

${ }^{31}$ BOSCO, Andrea, Federal Union e l'unione franco-britannica. Il dibattito federalista nel Regno Unito dal patto di Monaco al crollo della Francia, Bologna, Il Mulino, 2009, p. 49. 
sovrani $^{32}$. La formula del patto intergovernativo si rivelava incapace di strutturare un assetto europeo stabile e pacifico:

Ciò di cui vi era bisogno, concludevamo, non era una lega bensì un'assemblea eletta dagli abitanti degli Stati membri, che non solo potesse prendere decisioni per conto di tutti ma avesse anche l'autorità di renderle effettive33.

Ultimo ad unirsi al gruppo fu Patrick Ransome, giovane avvocato formatosi a Cambridge sotto la guida dello storico Harold Laski ${ }^{34}$, colui che diede a Federal Union una veste di "rispettabilità accademica" e che iniziò Rawnsley e Kimber alla teoria federalista. L'apporto di Ransome si rivelò determinante affinché il movimento elaborasse un programma a favore di un governo federale europeo, consentendo a Federal Union di conquistarsi un'identità originale e di emergere nell'agone sovraffollato delle associazioni pacifiste quale primo gruppo il cui scopo dichiarato era la creazione di una federazione delle democrazie europee. Significativo il fatto che, dopo l'arrivo di Ransome, il memorandum da cui l'organizzazione prendeva il nome cambiasse il titolo da Pax Union a Federal Union, una denominazione che marcava indelebilmente la militanza federalista del gruppo.

Secondo la ricostruzione offerta da Charles Kimber, nell'autunno del ' 38 si tenne l'incontro che sancì la nascita di Federal Union35. Alla riunione presenziarono circa ottanta persone e fu in quell'occasione che si decise di inviare il memorandum contenente i punti programmatici a cinquecento persone che nutrissero «interessi in affari istituzionali»36. I destinatari risposero entusiasti alla lettura del manifesto e, nel marzo del '39, venne indetta una seconda riunione per stabilire la strategia da adottare. All'incontro presenziarono, tra gli altri, la sociologa Barbara Wootton, docente di Studi Sociali all'Università di Londra, Wickham Steed, direttore del Times, nonché Lionel Curtis e Philip Kerr marchese di Lothian. Curtis e Kerr, ex funzionari dell'amministrazione coloniale, erano stati tra i principali sostenitori del federalismo imperiale, un progetto popolare nei circoli intellettuali inglesi negli anni Novanta dell'Ottocento, il quale si prefiggeva l'adozione di una struttura federale da parte

32 JOAD, Cyril, The Philosophy of Federal Union, in RANSOME, Patrick (ed.), Studies in Federal Planning, London, Lothian Foundation Press, 1990, p. 62.

33 KIMBER, Charles, «La Nascita di Federal Union», cit., p. 206.

34 BOSCO, Andrea, Federal Union e l'unione franco-britannica, cit., p. 50.

35 KIMBER, Charles, «La Nascita di Federal Union», cit., p. 208.

${ }^{36}$ Ibidem. 
dell'impero britannico ${ }^{37}$. Kerr in particolare si era dedicato, sul finire degli anni Venti, all'analisi del modello federale applicato a un contesto globale e i suoi scritti sul federalismo costituirono un'importante fonte di riflessione per i promotori di Federal Union.

Nel corso del '39, il nucleo di partecipanti elaborò un manifesto provvisorio, il quale si rivelava frutto di una riflessione europeista matura:

\begin{abstract}
National sovereignty leads to competition in armaments, economic self-sufficiency and internal regimentation, and thus inevitably to war, imperialism [...] and loss of individual liberty, because where sovereign States fail to agree there is no remedy save resort to violence. [...] No international order based on co-operation between sovereign States will prove either efficient or durable. [...] Nothing less than a union of the peoples can end this anarchy and give peace, justice, and freedom to all. Accordingly we advocate:

1. A Federal Union of those nations which hold that the State exists for the freedom and responsibility of man [...].

2. That this constitutional Union will [...] establish legislative, executive and judicial organs representative of and responsible to all the citizens of the Union for such common affairs as defence and order, currency, trade [...] and will possess the taxation and borrowing powers necessary to finance its own activities ${ }^{3}$.
\end{abstract}

Nel saggio The Philosophy of Federal Union, il filosofo Cyril Edwin Joad approfondì la concezione dello Stato sottesa al pensiero di Federal Union. Lo Statonazione, affermava, non era che una forma dell'organizzazione umana, lungi dall'esaurire o dal ridurre a sé tutte le altre. Immaginando un parallelismo tra l'evoluzione degli aggregati sociali e quella degli organismi biologici, Joad sottolineava che la tendenza del processo di sviluppo di entrambi è di aumentare le dimensioni «non della cellula o dell'individuo ma dell'unità di organizzazione»39. Così come le unità unicellulari si uniscono nel costituire un individuo che è un agglomerato di cellule, la società si forma per la progressiva associazione di corpi più piccoli. Perché, dunque, occorrerebbe accettare lo Stato-nazione come lo stadio ultimo, non transitorio, dell'organizzazione sociale umana e non supporre, piuttosto, una forma più ampia di governo data dall'unione tra queste macro-unità40? Da questo assunto partirono i lavori

\footnotetext{
37 BURGESS, Michael, The Federal Plan of the Imperial Federation League, 1892: Milestone or Tombstone?, in BOSCO, Andrea (ed.), The Federal Idea, cit., pp. 139-154.

${ }^{8}$ Citato in BURGESS, Michael, The British Tradition of Federalism, cit., p. 140.

39 JOAD, Cyril, op. cit., pp. 48-49.

40 Ibidem.
} 
dell'Istituto di Ricerca associato a Federal Union, presieduto da Ransome con la supervisione di William Beveridge, per conto del quale furono prodotti diversi lavori volti ad esaminare la teoria e la pratica dello Stato federale.

Un particolare lavoro prodotto per conto del Federal Union Research Institute si impone all'attenzione dell'osservatore, data la lucidità analitica con cui affronta la definizione del modello federale. Si tratta del saggio intitolato What Federal Government Is ad opera di Kenneth Wheare, costituzionalista in forza all'università di Oxford, un excursus breve ma efficace sulla natura del governo federale. Nell'incipit dell'analisi Wheare indagava le ragioni storiche che avevano fin lì dissuaso i britannici dall'intraprendere esperimenti federalisti di qualsiasi genere, sia rivolti all'organizzazione interna del proprio governo, sia rivolti ai rapporti con gli altri popoli d'Europa:

They are accustomed to a form of government one of the leading characteristic of which is that one single legislature, the King-in-Parliament at Westminster, has authority to make laws for the whole United Kingdom [...] and these laws duly made prevail over rules made by any other body in the Kingdom. [...] In a federation it is otherwise ${ }^{41}$.

Per quanto concerneva un'eventuale federazione europea, Wheare individuava due proprietà salienti per un governo sovranazionale dalle solide fondamenta: in primo luogo, una costituzione scritta, vincolante per tutte le autorità all'interno della federazione. Da essa «gli Stati e le autorità federali derivano i propri poteri, e ogni azione da questi compiuti in violazione ad essa risulta invalida» ${ }^{2}$. In secondo luogo, la federazione avrebbe dovuto disporre di risorse finanziarie autonome sia per il governo federale che i per i governi degli Stati federati. Se consideriamo che nel 1965 fu proprio il dibattito sulle risorse finanziarie autonome della CEE a scatenare una violenta crisi in seno alle istituzioni comunitarie (la cosiddetta crisi della sedia vuota43) è interessante

${ }^{41}$ WHEARE, Kenneth, What Federal Government Is, in RANSOME, Patrick (ed.), Studies in Federal Planning, cit., pp. 17-18.

${ }^{42}$ Ibidem, pp. 23-24.

43 L'episodio costituisce una delle più celebri controversie affrontate dalla Comunità Europea delle origini. Alla base dell'aspro dibattito stavano le visioni, diametralmente opposte, dell'innovatore Walter Hallstein, presidente della Commissione Europea, e Charles De Gaulle. Era nelle intenzioni di Hallstein fare della Commissione un vero e proprio esecutivo sovranazionale. Questo piano, di per sé bastevole a scatenare l'opposizione della Francia gollista (favorevole a un quadro comunitario che non compromettesse la superiorità dei governi nazionali rispetto agli organismi europei), si sposò con l'intenzione di Hallstein di dotare la CEE di autonome risorse finanziarie e di incrementare le competenze dell'Assemblea Parlamentare in materia di bilancio. La Francia si oppose e manifestò il proprio dissenso disertando le riunioni 
notare come Wheare, già negli anni Quaranta, avesse individuato la correlazione tra la maggiore autonomia in ambito finanziario del governo sovranazionale $\mathrm{e}$ l'indebolimento del controllo sugli organi federali da parte degli Stati nazionali.

Gli studi preparati per conto dell'Istituto di Ricerca fornirono un contributo indispensabile alla formulazione del modello federale europeo, riuscendo a renderlo straordinariamente popolare presso l'opinione pubblica britannica e internazionale ${ }^{44}$. I modelli di unione politica ed economica preparati da Federal Union costituirono difatti, come vedremo nelle conclusioni, una fonte di ispirazione primaria per quei gruppi attivi nell'Europa continentale che, soprattutto nel dopoguerra, si schierarono a favore di una federazione europea. Se il contributo teorico della cosiddetta "scuola federalista inglese" 45 ai progetti di integrazione sovranazionale fu determinante nel fondare una cultura federale europea, l'azione pratica di Federal Union non fu altrettanto incisiva, non riuscendo il movimento a influenzare concretamente l'azione del governo britannico. Chiamato a scegliere tra Europa e Atlantico, Federal Union si spaccò in due e al suo interno si aprì un dibattito destinato a inficiarne l'operato.

\section{I due volti di Federal Union: europeismo e atlantismo}

Nel rapido volgere degli anni che vanno dalla firma del Patto di Monaco alla battaglia di Londra, Federal Union registrò un notevole successo, forse troppo impetuoso perché potesse sedimentarsi e dare tempo al movimento di strutturarsi in una solida organizzazione. Federal Union conobbe una precoce nascita e una fine altrettanto rapida e tuttavia, nel giro di due anni, riuscì a suscitare il consenso tanto dei privati cittadini quanto degli esponenti della classe dirigente, conservatori o liberali che fossero. È perciò probabile che in assenza di un simile movimento il progetto per un'unione federale europea non avrebbe trovato in Gran Bretagna una rappresentanza altrettanto adeguata, come ebbe a sostenere fieramente Charles Kimber:

It is arguable that the British government's offer of union with France in 1940 would never have been made, nor Mr Attlee have declared that «Europe must federate or perish», had the movement not succeeded in getting the idea of federation so high on the agenda of public discussion with the support of so many

del Consiglio europeo. La crisi rientrò solo nel 1966, sanata dal compromesso di Lussemburgo. Cfr. MAMMARELLA, Giuseppe, Storia d'Europa dal 1945 a oggi, Roma, Bari, Editori Laterza, 2006, pp. 346-347.

44 BURGESS, Michael, The British Tradition of Federalism, cit., p. 147.

45 ROSSOLILLO, Francesco, La scuola federalista inglese, in ID., Senso della Storia e Azione Politica, vol. I, Bologna, Il Mulino, 2009, pp. 467-487. 
in high places ${ }^{4}$.

Nel momento in cui la minaccia del militarismo tedesco tornò a farsi concreta, Kimber, Ransome e Rawnsley sentirono che la generazione nata a ridosso del 1914 era chiamata a salvare l'Europa dal disastro. Questi giovani, che non avevano potuto prendere parte al primo conflitto mondiale, erano animati da sentimenti ambivalenti. Da una parte la delusione verso la generazione dei "padri", che si erano resi autori del Covenant della Società delle Nazioni senza tuttavia onorarne lo spirito:

Most put their faith in the Covenant of the League of Nations. [...] They have been naïve enough to have supposed that the old men who had remained in government form pre-war days had grown up in world of alliances and counter alliances and of competition for empire, had ever had the smallest intention of submitting to the terms of a Covenant which made war itself the only enemy47.

D'altra parte, essi nutrivano l'ambizione di creare un sistema europeo ex novo, alternativa pacifica all'integrazione del continente che si sarebbe realizzata altrimenti sotto la forza d'urto dell'invasione nazista. Federal Union si fece interprete di questa istanza di rinnovamento, eppure gli sforzi da essa profusi forse non sarebbero stati sufficienti se l'opinione pubblica non avesse avvertito l'incombere della guerra. Dalla lettura di autori protagonisti delle vicende federaliste ${ }^{48}$ si evince che, tra il 1937 e il 1939, fu proprio la coscienza della crisi della supremazia militare britannica a spingere il governo di Londra verso una politica estera meno isolazionistica, più propensa a ricercare contatti con l'Europa continentale e gli Stati Uniti. Fu dunque l'esaurirsi della Pax Britannica, già incrinata al tramonto dell'Ottocento dall'arrivo di nuove forze politiche - Stati Uniti e Germania in primis - e l'annesso timore per la sopravvivenza dell'impero a indurre parte della società inglese a ripensare i rapporti con l'Europa. A tal proposito il giornalista John Strachey commentò amaramente che il consenso suscitato dall'ipotesi europeista derivava dal fatto che gli inglesi, dopo essere rimasti per secoli fedeli ad una strategia di divide et impera nei confronti del continente erano ora disposti a tutto, anche a unirsi con Stati di cui avevano sempre diffidato, pur di

${ }^{46}$ KIMBER, Charles, Introduzione, in RANSOME, Patrick (ed.), Studies in Federal Planning, cit., p. V.

47 Ibidem, p. VI.

48 KERR, Philip, «The Political Situation in the United States», in Journal of the British Institute of International Affairs, 2, 4/1923, pp. 135-148 ; ID., "The Outlawry of War», in Journal of the Royal Institute of International Affairs, 7, 6/1928, pp. 361-388. 
salvare la patria 49 .

L'incapacità da parte di Federal Union di condizionare a lungo termine la vita politica non derivava solo dal fatto che l'ipotesi europeista era considerata da buona parte della società inglese un'extrema ratio, una scelta obbligata davanti alla minaccia di un nuovo, devastante, conflitto mondiale (sentimento ben espresso dal motto di Clement Attlee «federarsi o perire») ma anche da alcune contraddizioni intrinseche a Federal Union che i fondatori del movimento non riuscirono mai a risolvere del tutto. Si trattava di antinomie derivanti dal carattere eccezionale di Federal Union, dalla ossatura fluida, non gerarchizzata, adottata dal movimento ${ }^{50}$. Le ricostruzioni circa l'organizzazione di Federal Union indicano che quest'ultimo non ebbe mai la costituzione di un vero e proprio partito, prediligendo la forma di un movimento di opinione di massa intenzionato a calamitare tutti coloro che, a prescindere dal contesto politico di formazione e provenienza, si fossero detti favorevoli all'unione delle democrazie europee. Tale scelta ebbe come principale conseguenza il fatto di radunare individui provenienti da aree politiche spesso rivali, i quali portarono all'interno di Federal Union un universo di valori ideologici diversificati, che incisero immancabilmente sul modo di concepire la futura federazione europea.

La più grave di tali contraddizioni aveva natura "geografica" e ostacolò gravemente l'azione politica di Federal Union. Essa derivava dal fatto che all'interno del movimento coesistevano due diverse interpretazioni circa la federazione sovranazionale. La prima, di stampo europeista e sostenuta dai fondatori di Federal Union, riteneva che il nucleo territoriale della federazione avrebbe dovuto comprendere il solo continente europeo. La seconda, comune a molti simpatizzanti nonché a due federalisti della prima ora come Lionel Curtis e Philip Kerr, prediligeva l'idea di un'unione atlantica che comprendesse anche gli Stati Uniti, considerati il partner naturale della Gran Bretagna. Invero, l'idea di un duumvirato atlantico tra Gran Bretagna e Stati Uniti era diffusa, negli ambienti diplomatici inglesi, fin dal XIX secolo e trovava un nobile precursore nello storico Charles Dilke, tra i primi a marcare l'importanza della special relationship angloamericana per gli equilibri mondiali51. Il progetto per una federazione dal

49 BOSCO, Andrea, Federal Union e l'unione franco-britannica, cit., pp. 43-44.

5o MAYNE, Richard, PINDER, John, ROBERTS, John (ed.), Federal Union. The Pioneers, London, Palgrave Macmillan, 1990, p. 13.

${ }^{1}$ Cfr. ROBERTS, Priscilla, «Lord Lothian and the Atlantic World», in The Historian, 66, 1/2004, pp. 97-127; TAGLIAFERRI, Teodoro, L'idea di impero nella storiografia britannica del secondo $\mathrm{se}=10636 \&$ percorso $=/$ STORIA_DELLA_STORIOGRAFIA_CONTEMPORANEA\&idDocente $=54$ 454f444f524f5441474c4941464552524954474c54445236344330384638333943\&cognomeDoce nte=TAGLIAFERRI\&nomeDocente=TEODORO $>$ [consultato il 18 febbraio 2016] . 
baricentro anglosassone aveva riscosso un certo successo in seguito alla pubblicazione del pamphlet Union Now (1939) del giornalista americano Clarence Streit. Union Now suggeriva di istituire un dispositivo di sicurezza internazionale mediante un'unione federale di quindici democrazie occidentali (tra cui Stati Uniti, Gran Bretagna, Irlanda, Australia, Francia, Olanda). Le nazioni indicate come membri della federazione, argomentava Streit, costituivano un blocco politico e finanziario formidabile, con l'autorità necessaria per mantenere la pace senza ricorrere alla guerra. Venivano poi individuate alcune aree strategiche da unificare sotto la competenza dell'Unione a venire, ovvero cittadinanza, difesa e politica monetaria. Si trattava, insomma, di replicare lo spirito dell'impresa federale americana in terra europea:

I mean that we the people of the United Democracies transfer these five power from our fifteen national governments to a union government, just as we the people of the United States transferred these same powers to a union of thirteen states in $1878^{52}$.

In Inghilterra Union Now fu pubblicato grazie agli sforzi di Lionel Curtis che lo inviò, tra gli altri, a Robert Cecil, Anthony Eden e Winston Churchill53. Il fatto che Union Now fosse stato praticamente concomitante con la nascita di Federal Union non deve indurre a una sovrapposizione lineare tra i due progetti. I fondatori di Federal Union conoscevano il testo di Streit, tanto che Ransome ammise di essere rimasto impressionato dalla precisione argomentativa dell'americano54. Tuttavia Union Now pur rappresentando un testo influente non determinò la creazione del movimento55, né tantomeno suggestionò l'idea di federazione che i tre fondatori avevano in mente. Nelle intenzioni di Streit preludio necessario alla costruzione della federazione sovranazionale era l'unione di Gran Bretagna e Stati Uniti e non è casuale perciò che Streit intrattenesse una fitta corrispondenza più con Curtis e Kerr (rappresentanti di spicco dell'ala atlantista) che non con i fondatori di Federal Union ${ }^{56}$. Il progetto di unione atlantica enucleato nella draft constitution contenuta in Union Now, ricalcava da vicino la Carta americana e apparve, a Kimber e a Ransome, come un tentativo di trasporre forzatamente il modello federale americano al composito gruppo di Stati

${ }^{2}$ STREIT, Clarence, "The Atlantic Union Plan and the Americas», in The Annals of the American Academy of Political and Social Science, 204, 1/1939, pp. 93-101.

53 BOSCO, Andrea, Federal Union e l'unione franco-britannica, cit., p. 32.

54 ID., «Lothian, Curtis, Kimber and the Federal Union Movement», in Journal of Contemporary History, 23, 3/1988, pp. 465-503.

55 CURRY, William Burnely, The Case for Federal Union, London, Penguin, 1939, p. 19.

${ }^{6}$ CASTELLI, Alberto, Una pace da costruire: $i$ socialisti britannici e il federalismo, Milano, Franco Angeli, 2002, pp. 76-77. 
europei. Una federazione di tal genere non sembrava loro né realizzabile né tantomeno desiderabile. Perciò essi decisero di dare alla propria battaglia per la federazione una connotazione europeista: l'Europa, il continente che deteneva le "chiavi della guerra e della pace”, avrebbe dovuto essere il vero centro della federazione di Stati anziché la semplice componente periferica di un'unione anglo-americana. Kimber spiegò successivamente le differenze che correvano tra il progetto europeista dei fondatori e quello atlantista. Pur trattandosi di proposte ispirate a una medesima teoria politica, esse erano in disaccordo sulla base geografica della federazione:

Come ho già detto pensavamo solo alle democrazie europee. [...] Prima di aver avuto il tempo di elaborare questo concetto, fummo superati dalla vasta pubblicità ottenuta da Union Now di Clarence Streit. [...] Esso contribuiva molto validamente a sostenere la tesi della federazione in quanto contrapposta alla lega; ma come proposta politica sembrò a noi che eravamo i tre iniziatori di Federal Union alquanto irrealistica e [...] certamente non auspicabile: noi eravamo europei57.

Nel corso della fase organizzativa del movimento, nella primavera del 1939, il dualismo atlantismo-europeismo era destinato a riemergere, impedendo l'individuazione di una strategia coerente capace di tenere unito un movimento così atipico. Lo scoppio della guerra rese necessario un'esplicita dichiarazione di intenti da parte di Federal Union e, sebbene Kimber annunciasse che Federal Union si sarebbe battuta per un'unione delle sole democrazie europee, la natura ultima della federazione rimase di fatto indefinita. Aspetti importanti quali il nucleo territoriale e il sistema di rappresentanza parlamentare da adottare (bicamerale o monocamerale) furono deferiti a un imprecisato futuro e, alla fine, mai dibattuti. Il compromesso tra atlantisti ed europeisti, probabilmente frutto del tentativo di non disperdere fin da subito le forze del neonato movimento, finì con l'annacquare lo spirito originario che aveva portato alla nascita di Federal Union e, sulla lunga distanza, ne paralizzò l'azione a livello istituzionale rendendola meno coesa e provocando la perdita di molti sostenitori ${ }^{5}$. Con l'infiammarsi del conflitto le difficoltà di Federal Union crebbero esponenzialmente. Il movimento, all'apice della popolarità tra il 1938-40, si avviò verso la sua parabola discendente già nel 1940. Le sezioni distaccate registrarono un netto declino nella partecipazione mentre la propaganda sul territorio si fece stentata, per carenza di fondi e personale 59 . In aggiunta, il nucleo originario dei fondatori si era dissolto o per la

57 KIMBER, Charles, «La Nascita di Federal Union», cit., pp. 208-209.

$5^{8}$ BOSCO, Andrea, Federal Union e l'unione franco-britannica, cit., p. 72.

59 MAYNE, Richard, PINDER, John, ROBERTS, John (ed.), op. cit., p. 33. 
chiamata al fronte (Rawnsley) o in seguito alle dimissioni dovute a un aspro dibattito tra le correnti interne del movimento (Kimber). L'entusiasmo suscitato dal federalismo si preparava ad essere eclissato prima dall'anti-europeismo del governo laburista e poi dalla strategia confederale del governo conservatore di Churchill.

\section{Conclusioni}

$\mathrm{Al}$ termine di questa rapida rassegna, possiamo individuare alcuni tratti significativi nella storia delle relazioni tra la Gran Bretagna e l'integrazione europea. La cultura anglosassone tra XIX e XX secolo è percorsa da una forte corrente federalista rappresentata da personalità di spicco come John Seeley, Philip Kerr, William Beveridge. La comparsa di Federal Union segnò poi «lo spartiacque tra una forma arcaica e una matura di federalismo» ${ }^{60}$, dando un duplice apporto alla causa europeista. In primo luogo esso contribuì allo sviluppo di quella che, prendendo il termine in prestito da Federico Chabod ${ }^{61}$, possiamo definire la coscienza di essere europei. Nel momento in cui l'Europa cedeva sotto la pressione divisoria del nazionalismo Federal Union operò una pubblicizzazione indefessa dell'unità politica e culturale degli europei. In secondo luogo, Federal Union capì che «la pace non è frutto di buone intenzioni bensì deriva da un effettivo ordinamento degli affari comuni dell'umanità»62 e coniugò la prassi alla teoria, cercando di agire nel dibattito politico per indirizzare l'azione delle forze governative in senso filo-europeista.

Occorrerà notare tuttavia che il federalismo raggiunse il suo apice in corrispondenza di forti alterazioni degli equilibri continentali che minacciavano l'egemonia britannica. Si può dunque individuare una relazione funzionale tra l'aumento di popolarità dell'idea europeista e il ridimensionamento geopolitico della Gran Bretagna, in virtù della quale la soluzione federalista venne raramente considerata nella propria autonomia e piuttosto utilizzata come risposta estemporanea a periodi di crisi localizzati, superati i quali si poteva ripiegare dietro i baluardi tradizionali della sovranità e dell'impero. Quanto quest'ultimo fosse ancora al centro della narrazione storica prediletta dai britannici (anche coloro che più erano sensibili ai problemi della pace e dell'Europa) lo si evince dal fatto che uno dei più esaustivi progetti di costituzione federale, elaborato dal giurista Ivor Jennings per conto del Constitutional Committee di Federal Union, annoverava quali membri della

6o BOSCO, Andrea, Federal Union e l'unione franco-britannica, cit., p. 435.

${ }^{61}$ CHABOD, Federico, Storia dell'Idea di Europa, Roma, Bari, Editori Laterza, 1989, p. 13.

62 CURRY, William Burnely, op. cit., p. 20. 
federazione sovranazionale non solo le democrazie europee ma anche i dominion britannici63. Il modello federale appare qui come soluzione volta a coniugare dimensione imperiale e dimensione comunitaria europea, capace di generare un'entità politica di formidabile estensione (comprendente l'Europa e le propaggini coloniali britanniche). Una federazione così concepita si dimostrava congeniale nel conservare il ruolo e l'immagine della Gran Bretagna quale grande potenza. Di fatto, si trattava di un disegno destinato a non realizzarsi. Fu anzi l'attaccamento alla passata grandezza imperiale che indusse la Gran Bretagna a sostenere, negli anni di formazione della CEE, un tipo di cooperazione intergovernativa meno innovativa rispetto a quella suggerita da Federal Union e più cauta rispetto ai progetti che venivano avanzati in altri ambienti europei, federalisti o funzionalisti che fossero.

Federal Union resta caso paradigmatico di una ricca tradizione di pensiero europeista a cui la Gran Bretagna avrebbe potuto guardare per svolgere un ruolo più attivo nel processo di integrazione europea post-bellico. Un processo che, almeno in parte, fu ispirato proprio dagli scritti dei federalisti inglesi i quali influenzarono l'azione degli epigoni continentali (italiani in particolare ${ }^{64}$ ) che presero parte alla costruzione comunitaria nel dopoguerra. Federal Union costituisce dunque un valido modello da analizzare qualora si volesse tentare di risolvere il quesito, posto a suo tempo da Charles Kimber, se i britannici siano davvero europei o siano parte di un "mondo separato di lingua inglese".

63 JENNINGS, Ivor, Rough Draft of a proposed Constitution for a Federation of Western Europe, in RANSOME, Patrick (ed.), Towards the United States of Europe. Studies on the Making of European Constitution, London, Lothian Foundation Press, 1991, p. 138.

${ }^{64} \mathrm{La}$ tradizione culturale inglese influenzò quanti, in Italia, si pronunciarono a favore di una sovranità sovra-statale. In sede critica si è evidenziato come, ad esempio, il pensiero di Locke e Hamilton abbia ispirato la riflessione di Luigi Einaudi, Attilio Cabiati e Giovanni Agnelli i quali, sul finire degli anni Dieci del XX secolo, elaborarono testi di riflessione sul federalismo e sulla limitazione del potere sovrano. Cfr. MALANDRINO, Corrado, Federalismo. Storia, idee, modelli, Roma, Carocci Editore, 1998, p. 99. Lo stesso Altiero Spinelli riconobbe in autori come Philip Kerr e Lionel Robbins dei maestri, affermando che: «[la mia] attenzione non fu attratta dal fumoso e contorto federalismo ideologico di tipo proudhoniano o mazziniano, ma dal pensiero pulito e preciso di questi federalisti inglesi, nei cui scritti trovai un metodo assai buono per analizzare la situazione nella quale l'Europa stava precipitando», cfr. SPINELLI, Altiero, Come ho tentato di diventare saggio. Io, Ulisse, Bologna, Il Mulino, 2006, pp. 307-308; PINDER, John, «Il federalismo in Gran Bretagna e in Italia: i radicali e la tradizione liberale inglese», in The Federalist, 2/1989, p. 92 et seq. 


\section{* L'autore}

Benedetta Giuliani ha conseguito nel 2014 la laurea in Storia, Antropologia e Religioni presso l'Università degli Studi di Roma "La Sapienza", discutendo una tesi sul rapporto tra la Gran Bretagna e l'integrazione europea. Ha collaborato con la rivista «Eurostudium». Attualmente è iscritta alla laurea magistrale in Scienze Storiche presso l'università di Roma "La Sapienza".

URL: < http://www.studistorici.com/progett/autori/\#Giuliani >

\section{Per citare questo articolo:}

GIULIANI, Benedetta, "Federarsi o perire". II contributo britannico al federalismo europeo», Diacronie. Studi di Storia Contemporanea : "Se creare è definire", 29/03/2016,

URL:< http://www.studistorici.com/2016/03/29/giuliani_numero_25/ >

Diacronie Studi di Storia Contemporanea $\vartheta$ www.diacronie.it

Risorsa digitale indipendente a carattere storiografico. Uscita trimestrale. redazione.diacronie@hotmail.it

Comitato di redazione: Jacopo Bassi - Luca Bufarale - Elisa Grandi - Antonio César Moreno Cantano - Deborah Paci - Fausto Pietrancosta - Alessandro Salvador - Matteo Tomasoni - Luca Zuccolo

Diritti: gli articoli di Diacronie. Studi di Storia Contemporanea sono pubblicati sotto licenza Creative Commons 3.0. Possono essere riprodotti e modificati a patto di indicare eventuali modifiche dei contenuti, di riconoscere la paternità dell'opera e di condividerla allo stesso modo. La citazione di estratti è comunque sempre autorizzata, nei limiti previsti dalla legge. 